\title{
WCC 2016-119 : Role of Speckle Tracking Echocardiography In Noninvasive Assessment Of Pulmonary Vascular Resistance In Patients With Pulmonary Hypertension.
}

\author{
K.V.Siva Krishna, B.Srinivas, M.Jyotsna, D.SeshaGiriRao \\ Background:
}

Assessment of Pulmonary Vascular Resistance in patients with Pulmonary Arterial Hypertension plays important role in the prognosis of these patients. The gold standard investigation in the assessment of PVR is cardiac catheterisation. Noninvasive estimate of PVR is essential both for diagnosis, prognosis and follow up of these patients. The Doppler derived ratio of TRV/TVI $\mathrm{RVOT}_{\mathrm{R}}$ correlated well with invasive PVR in several studies. But the draw backs are lack of correlation in high PVR situations (PVR > $6 \mathrm{WU}$ ), and inadequacy of obtaining an adequate TRV spectrum in some patients. 2D strain measurements are independent of acquisition angle and preload status and RV longitudinal strain has recently been shown to correlate well RV function and PAP as well as survival in subjects with pulmonary arterial hypertension (PAH). It is hypothesized that RV strain measurements would be more readily obtained than estimates of PAP from tricuspid regurgitation (TR) and that reduced absolute values of RV strain would correlate with invasive hemodynamic measurements indicative of PH such as pulmonary vascular resistance (PVR).

\section{Materials and methods:}

In the present study 30 patients with PAH both congenital and acquired, and PVR >6 wu were studied retrospectively. In all these patients RV basal systolic strain is calculated by speckle track imaging within 8 hours of cardiac catherisation. Patients with PVR $<6$ WU were excluded from the study.

\section{Results:}

The Study group consisted of 30 patients of age between 1 and 60 years. The mean age of study population is $29.1+14.9$. males constituted $33 \%$ of study population. The mean pulse rate is 95 +14.76 , the mean PAP was $59.83+10.72$. About $60 \%$ of patients were in functional class 2 at the time of recruitment in the study. The most common diagnosis in these patients is primary pulmonary hypertension, followed by Ostium Secundum ASD with severe PAH. The mean PVR calculated by catheterisation in the study population was 15.08. Linear regression analysis done between RV systolic strain, and PVRcath and a regression equation was generated. The echo derived parameter showed a good negative correlation with invasive cath with $\mathrm{r}=-0.81, \mathrm{p}=$ 
$<0.0001$. regression equation was generated to calculate PVR from RVSS. Bland altmann Analysis showed neglible bias between the two methods with 95\% confidence limits being -7.21 and 7.21.

\section{Conclusion:}

RVSS is a reliable non invasive parameter for estimation of PVR by echocardiography in patients with high PVR and PAH and in those with inadequate and unreliable TR jet velocities. This investigation can be used in the follow up assessment of PVR in these individuals to track changes in PVR. 